$\xi=\mathbf{Z}$

\title{
Origin of dark mass apparent from gravitationally bound extended ordinary material systems
}

\author{
Shubhen Biswas * \\ G.P.S.H.School Alaipur, Nadia, Pin-741245(W.B.), India \\ *Corresponding author E-mail: shubhen3@gmail.com
}

\begin{abstract}
To grow up a gravitationally bound system with fixed proper mass requires sufficient energy to overcome the gravitational pull. Following General relativity both matter energy and field energy act as the sources of gravity. Apart from the field energy if our concern is just for the bound system of mass only, then we can easily attribute the gain of matter energy as the gain of gravitational mass. This gain of matter energy can be backed immediately if the system is allowed to collapse to its initial state. So in the case of expansion there will be no possibility in creation of normal or proper mass. But the concern regarding the gain of matter energy must have realistic effect in increase of gravitational mass. This article explains how gravitational mass of the gravitationally bound large material system like galaxy exceeds its ordinary mass with the size of the system. Here presence of the dark mass and flat rotation curve are given without considering MOND theory, not even distorting any accepted paradigm of post Newtonian gravity. The findings are truly consistent with the recent observed data.
\end{abstract}

Keywords: Gravitation; Matter Energy; Binding Energy; Relativistic Process; Dark Mass.

\section{Introduction}

In explaining the mass of a galaxy cluster using virial theorem [7], Zwicky [20] first observed cluster mass is high enough over the luminous matter. Following Vera Rubin et al [16] luminous ordinary matter in our galaxy is not sufficient to describe the flat rotation curve of the stars outskirt the galaxy disk, it requires large amount of additional mass. These entire events then require a hypothetical hidden mass responsible for unseen dark matter, which prevails throughout the whole universe. But still now direct searches don't have revealed any presence of the dark matter. And several theoretical approaches [5] have been made to explain the alternative of dark matter, the most one of them Modified Newtonian Dynamics or simply MOND by Milgrom [12] but have some discrepancies [3].So by the way for any gravitationally bound system, whether it is galaxy or galaxy clusters there always have certain amount of measurable dark matter.

But gravity of a material system is the effect of systems matter energy in the general relativistic concept [2][18] so in consequence of gravitational mass of a collection of large extended gravitationally bound system of ordinary matter our concern will be pointed only over the matter energy of the entire system.

Now total energy of a gravitationally bound system can be given as [10]

$\mathrm{E}=\mathrm{E}_{\mathrm{M}}+\mathrm{E}_{\mathrm{F}}$

From energy momentum tensor $\mathrm{T}_{0}^{0}$ in classical limit matter energy

$E_{M}=\int T_{0}^{0} \sqrt{-g} d^{3} r=\int \rho\left(c^{2}+\frac{1}{2} v^{2}-\varphi\right) d^{3} r$
And field energy

$\mathrm{E}_{\mathrm{F}}=\frac{1}{8 \pi \mathrm{G}} \int|\nabla \varphi|^{2} \mathrm{~d}^{3} \mathrm{r}$

Where $\varphi=\sum \frac{\mathrm{GM}}{\mathrm{r}}$, Newtonian gravitational potential

The source of gravity is presumably the matter energy that is gravitational mass or inertial mass can be inferred apart from field energy [13]. Hence any gain or loss of mass for a gravitationally bound system can only be interpreted in terms of matter energy.

Now let us have a simple gravitationally bound system which can be achieved by considering a central mass ' $\mathrm{M}_{0}$ ' and a ring of thin layer of baryonic mass ' $m$ ' orbiting with speed ' $v$ ' concentric to the central mass keeping a radial distance ' $r$ ' such that under force equilibrium mass of the ring will not fall inwards and maintain the fixed radial distance.

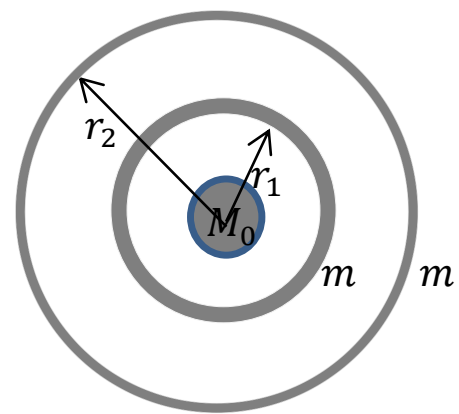

Fig. 1: Gravitationally Bound Ring of Ordinary Mass. 
Implying these in equation (2) total matter energy of the gravitationally bound system

$$
E_{M}=m^{2}-\frac{G_{0} m}{2 r}+P V+\varepsilon_{M_{0}}
$$

Now keeping the total baryonic mass fixed, if the orbit of the thin layer is stretched from $r_{1}$ to $r_{2}$ isothermally such that the pressure energy PV of the lamina and the assumed matter energy of the central mass $\varepsilon_{\mathrm{M}_{0}}$ remain unchanged, then the system requires certain amount of additional energy just to elevate the energy state.

From equation (4) the increase in matter energy for transforming $1^{\text {st }}$ to the $2^{\text {nd }}$ state with the condition, the pressure energy remains constant we have

$$
\Delta \mathrm{E}_{\mathrm{M}}=\frac{1}{2} \mathrm{GM}_{0} \mathrm{~m}\left(\frac{1}{\mathrm{r}_{1}}-\frac{1}{\mathrm{r}_{2}}\right)
$$

The gain of matter energy from equation (5) is just what that is the difference between their gravitational binding energy. Ultimately this matter energy enhances the gravity thus the system becomes more gravitationally massive. Corresponding gain in mass for the $2^{\text {nd }}$ relative to the converged System

$$
\Delta \mathrm{M}=\frac{\Delta \mathrm{E}_{\mathrm{M}}}{\mathrm{c}^{2}}
$$

Using equation (5) $\Delta \mathrm{M}=\frac{1}{2} \frac{\mathrm{GM}_{0} \mathrm{~m}}{\mathrm{c}^{2}}\left(\frac{1}{\mathrm{r}_{1}}-\frac{1}{\mathrm{r}_{2}}\right)$

Equation (6) reveals to grow up in size [Fig-1] the system needs additional energy, which ultimately appears in equivalent amount of additional mass. This gain of energy can be backed immediately if the system is allowed to collapse in on-self. So in this case the process of gain in mass will restrict the possibility in creation of ordinary matter. Hence at the expansion gravitationally bound system is proposed to be gravitationally massive over the total baryonic mass by achieving electromagnetically non interacting mass.

\section{The binding energy for galaxy}

The preceding section deals with the gain of hidden mass for gravitationally bound system. The laboratory based system for its lower mass doesn't possess enough matter energy to create a measurable hidden mass. Since origin of hidden mass is quadratic to the ordinary mass and increases linearly with the increasing of size so in creating large amount of hidden mass we need to look for a massive vastly rarified gravitationally bound system. Observations show [4] Galaxy is a vast highly rarified system with enormous amount of luminous matter like stars and gases, which are gravitationally bound with a central black hole. Thus following equation (6) there is the possibility to find out larger amount of confined hidden mass over the ordinary mass.

Let us have a disc galaxy [Fig 2] of radii $r_{g}$ made off having gravitationally bound total baryonic matter of mass $M$ with the central black hole of mass' $\mathrm{M}_{0}{ }^{\prime}$. If the disk mass $\left(M-M_{0}\right)$ was gravitationally squeezed to concentrate at the galactic center within a radius $r_{0}$ at the vicinity surrounding the central black hole then this system required sufficient mechanical energy to expand the whole system occupying large galactic size. Hence these two systems one is condensed and other is highly rarified the true galaxy, are not identical but differ by large amount of matter energy due to their gravitational binding energy even they have equal baryonic mass.

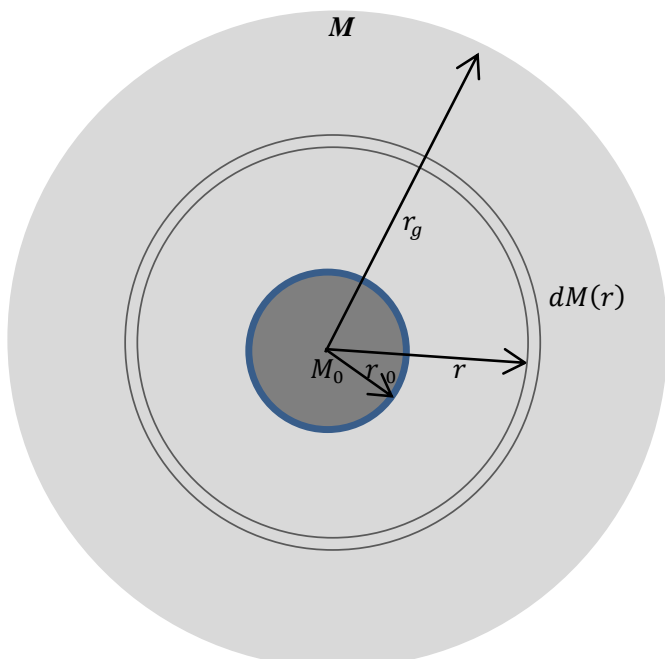

Fig. 2: Galaxy Disc as a Gravitationally Bound Rarified Ordinary Matter Distribution.

In a large scale the stealer objects and interstellar gasses of galaxy can be treated as a continuous mass distribution and the gravitational binding energy of the disc mass within range of radii $\mathrm{r}_{0} \rightarrow \mathrm{r}_{\mathrm{g}}[$ Fig-2]

$E_{g}=\frac{1}{2} G \int_{r_{0}}^{r_{g}} \frac{M(r) d M(r)}{r}$

Similarly gravitational binding energy for the concentrated $\operatorname{mass}\left(\mathrm{M}-\mathrm{M}_{0}\right)$ in orbital radius $\mathrm{r}_{0}$ outside the event horizon of the central black hole

$E_{c}=\frac{1}{2} G \int_{0}^{\left(M-M_{0}\right)} \frac{\left(M_{0}+m\right) d m}{r_{0}}$

Then stored matter energy within the galaxy relative to the compact system

$\Delta \mathrm{E}_{\mathrm{M}}=\mathrm{E}_{\mathrm{c}}-\mathrm{E}_{\mathrm{g}}$

$\Delta \mathrm{E}_{\mathrm{M}}=$

$\left[\frac{1}{2} G \int_{0}^{\left(M-M_{0}\right)} \frac{\left(M_{0}+m\right) d m}{r_{0}}\right]_{\text {Compact orbit }}-$

$\left[\frac{1}{2} \mathrm{G} \int_{\mathrm{r}_{0}}^{\mathrm{rg}} \frac{\mathrm{M}(\mathrm{r}) \mathrm{dM}(\mathrm{r})}{\mathrm{r}}\right]_{\text {Galaxy disc }}$

\section{The radial distribution of luminous mass for a disc galaxy}

For galaxy the direct measurement of luminous mass reveals a radial decline of matter density from the end of central bulge. Hence a reasonable simulation of the density distribution in decreasing fashion $\rho(r) \sim r^{-\alpha}$, could make it simple to calculate the second integral in the square bracket.

For sb type disc galaxy applying power law

$\rho(r)=\rho_{0}\left(\frac{r}{\sigma}\right)^{-\alpha}, \sigma$ is the scaling factor

$\rho(r)=K r^{-\alpha}$, here constant $K=\rho_{0} \sigma^{\alpha}$

The corresponding mass of the disc with radius $r$ will be

$$
\begin{aligned}
& M(r)=M_{0}+\int_{r_{0}}^{r} 2 \pi r \rho(r) d r \\
& M(r)=M_{0}-\frac{2 \pi K}{\alpha-2}\left(r^{-\alpha+2}-r_{0}^{-\alpha+2}\right)
\end{aligned}
$$


The equation restricts not to choose value of alpha arbitrarily, if $\alpha=1$ mass of the galactic disk increases linearly with radial distance $r$ and for greater than 2 it decreases with $r$ which is absurd but both the situations are not allowed by the observations of luminous mass distribution. So condition $1<\alpha<2$ will be suitable to describe the mass distribution of a disc galaxy.

A suitable choice for $\alpha=1.5$ leads $K=\frac{M-M_{0}}{4 \pi\left(r_{g^{\frac{1}{2}}}-r_{0} \frac{1}{2}\right)}$

\section{The gravitational binding energy of the gal- axy}

The whole galaxy can be treated as an extended continuous mass distribution, hence the integral part for total binding energy of the galaxy following equations

$\int_{r_{0}}^{r_{g}} \frac{M(r) d M(r)}{r}=$
$M_{0} \int_{r_{0}}^{r_{g}} \frac{d M(r)}{r}+\frac{2 \pi K r_{0}-\alpha+2}{\alpha-2} \int_{r_{0}}^{r_{g}} \frac{d M(r)}{r}-\frac{2 \pi K}{\alpha-2} \int_{r_{0}}^{r_{g}} \frac{r^{-\alpha+2} d M(r)}{r}$

Further from equation (13) $\frac{d M(r)}{r}=\left(2 \pi K r^{-\alpha}\right) d r$

Using this in equation (15)

$\int_{r_{0}}^{r_{g}} \frac{M(r) d M(r)}{r}=$

$2 \pi K M_{0} \int_{r_{0}}^{r_{g}} r^{-\alpha} d r+$

$\frac{(2 \pi K)^{2} r_{0}^{-\alpha+2}}{\alpha-2} \int_{r_{0}}^{r_{g}} r^{-\alpha} d r-\frac{(2 \pi K)^{2}}{\alpha-2} \int_{r_{0}}^{r_{g}} r^{-2 \alpha+2} d r$

Since our choice of $\alpha=\frac{3}{2}$ and using equation (14)

$1^{\text {st }}$ term of RHS equation (16)

$2 \pi K M_{0} \int_{r_{0}}^{r_{g}} r^{-\frac{3}{2}} d r=-\frac{\left(M-M_{0}\right)}{\left(r_{g}^{\frac{1}{2}}-r_{0} \frac{1}{2}\right)}\left(r_{g}^{-\frac{1}{2}}-r_{0}^{-\frac{1}{2}}\right)$

But for galactic disc $r_{g} \gg r_{0} 1^{\text {st }}$ term reduces to

$2 \pi K M_{0} \int_{r_{0}}^{r_{g}} r^{-\frac{3}{2}} d r=\frac{\left(M-M_{0}\right) M_{0}}{r_{g^{\frac{1}{2}} r_{0} \frac{1}{2}}}$

Likewise $2^{\text {nd }}$ term,

$\frac{(2 \pi K)^{2} r_{0}^{\frac{1}{2}}}{\alpha-2} \int_{r_{0}}^{r_{g}} r^{-\frac{3}{2}} d r=\frac{\left(M-M_{0}\right)^{2}}{r_{g}}$

$3^{\text {rd }}$ term,

$\frac{(2 \pi K)^{2}}{\alpha-2} \int_{r_{0}}^{r_{g}} r^{-1} d r=-\frac{\left(M-M_{0}\right)^{2}}{2 r_{g}} \ln \frac{r_{g}}{r_{0}}$

Thus for a disc galaxy gravitational binding energy responsible for total component of rarified baryonic mass will be

$\frac{1}{2} G\left[\int_{r_{0}}^{r_{g}} \frac{M(r) d M(r)}{r}\right]=G\left[\begin{array}{c}\frac{\left(M-M_{0}\right) M_{0}}{2 r_{g}^{\frac{1}{2}} r_{0}^{\frac{1}{2}}}+\frac{\left(M-M_{0}\right)^{2}}{2 r_{g}} \\ +\frac{\left(M-M_{0}\right)^{2}}{4 r_{g}} \ln \frac{r_{g}}{r_{0}}\end{array}\right]$

Further if the total baryonic mass of the galaxy excluding central black hole mass was assumed to be concentrated in a small orbit of radius $r_{0}$ just outside the black hole event horizon under strong gravity then gravitational binding energy of the condensed system

$\frac{1}{2} G \int_{0}^{\left(M-M_{0}\right)} \frac{\left(M_{0}+m\right) d m}{r_{0}}=G\left[\frac{\left(M-M_{0}\right)^{2}}{4 r_{0}}+\frac{\left(M-M_{0}\right) M_{0}}{2 r_{0}}\right]$

Since gravitational binding energy at the vicinity of black hole may exceed rest mass energy by Bardeen et al [1] then equation (22) must not be contradictory at this point.

\section{Invisible mass for a disc galaxy}

From sections it is obvious there is a huge difference between the two systems even they have equal baryonic mass. The first system that is responsible for galaxy itself will have an amount of gravitational binding energy in excess of the second system. The amount of stored gravitational energy from equations (10), (21) and (22)

$\Delta E_{M}=G\left[\begin{array}{c}\frac{\left(M-M_{0}\right)^{2}}{4 r_{0}} \\ +\frac{\left(M-M_{0}\right) M_{0}}{2 r_{0}}\end{array}\right]-G\left[\begin{array}{c}\frac{\left(M-M_{0}\right) M_{0}}{2 r_{g}^{\frac{1}{2}} r_{0}^{\frac{1}{2}}} \\ +\frac{\left(M-M_{0}\right)^{2}}{2 r_{g}}+\frac{\left(M-M_{0}\right)^{2}}{4 r_{g}} \ln \frac{r_{g}}{r_{0}}\end{array}\right]$

Introducing $\left(M-M_{0}\right)=M_{B}$ as the total galactic Baryonic mass for ordinary matter

$\Delta M=\frac{G}{c^{2}}\left[\frac{M_{B}^{2}}{4 r_{0}}+\frac{M_{B} M_{0}}{2 r_{0}}-\frac{M_{B} M_{0}}{2 r_{g}{ }^{\frac{1}{2}} r_{0} \frac{1}{2}}-\frac{M_{B}^{2}}{2 r_{g}}-\frac{M_{B}^{2}}{4 r_{g}} \ln \frac{r_{g}}{r_{0}}\right]$

For Milky Way disc galaxy within the solar circle yields a total baryonic mass for the Milky Way of around $6.1 \pm 0.5 \times$ $10^{10} M_{\odot}$ of which $4.9 \pm 0.4 \times 10^{10} M_{\odot}$ lies within the solar circle 2-5kpc[11] also total stellar mass of $6.43 \pm 0.63 \times$ $10^{10} M_{\odot}$ within solar orbit range $\left(r_{g}\right) \sim 8.29 \mathrm{kpc}$ by McMillan [9]. From radio source Milky Way central black hole Sgr A* measured radius $\left(r_{0}\right) \sim 9 A U$ [8] [9] and calculated $M_{0} \sim 3.7 \pm 1.5 \times$ $10^{6} M_{\odot}[16]$ [6].

Hence choosing in equation (24)

$M_{0} \sim 4 \times 10^{6} M_{\odot}, M_{B} \sim 6.5 \times 10^{10} M_{\odot}$

$r_{0} \sim 9 A U, r_{g} \sim 8.29 k p c$

Where solar mass $M_{\odot} \sim 1.9884 \times 10^{30} \mathrm{~kg}$ and $\frac{\mathrm{G}}{\mathrm{c}^{2}} \sim 7.42 \times \frac{10^{-28} \mathrm{~m}}{\mathrm{~kg}}$ The square bracket of equation (24) all the terms is negligible comparing to the $1^{\text {st }}$ term.

$\Delta M \sim \frac{G M_{B}{ }^{2}}{4 c^{2} r_{0}}$

Remarkably this large amount of mass and its non-interacting property unlike ordinary matter in our galaxy can be compared with hypothetical dark matter.

\section{Results}

i) Equation (25) gives amount of hidden mass $\Delta \mathrm{M} \sim 1.162 \times$ $10^{12} \mathrm{M}_{\odot}$

Thus total gravitational mass or virial mass comes to be

$(\mathrm{M}+\Delta \mathrm{M})=\mathrm{M}_{0}+\mathrm{M}_{\mathrm{B}}+\frac{\mathrm{GM}_{\mathrm{B}}^{2}}{4 \mathrm{c}^{2} \mathrm{r}_{0}}$

Following equation (26) total virial mass becomes $\mathrm{M}_{\mathrm{v}} \sim 1.23 \times$ $10^{12} \mathrm{M}_{\odot}$, this value is truly comparable to the obtained virial mass $\mathrm{M}_{\mathrm{v}} \sim 1.26 \pm 0.24 \times 10^{12} \mathrm{M}_{\odot}$ by McMillan [9] of the host galaxy inside suns orbital range $\mathrm{r}_{\mathrm{g}} \sim 8.29 \mathrm{kpc}$, shares almost $\frac{\Delta \mathrm{M}}{\mathrm{M}+\Delta \mathrm{M}} \geq$ $90 \%$ to the total gravitational mass or virial mass.

(ii) From equations (13) and (14) radial ordinary mass distribution, $M_{B}(r) \propto r^{1 / 2}$, implying in equation (25) we have almost constant radial mass density as expected from flat rotation curve [15].

(iii)The all planets share ordinary mass (excluding the sun) $\mathrm{M}_{\mathrm{B}} \sim 2.658 \times 10^{27} \mathrm{~kg}$ which is 1000 times less than solar mass $\mathrm{M}_{\odot} \sim 1.9884 \times 10^{30} \mathrm{~kg}$, now allowing the total planetary mass in a compact orbit of solar radius $R_{\odot}=6.963 \times 10^{8} \mathrm{~m}$ the 
amount of hidden mass using the leading term $\frac{\mathrm{GM}_{\mathrm{B}} \mathrm{M}_{0}}{2 \mathrm{c}^{2} \mathrm{r}_{0}}$ of equation (24)gives $\sim 2.8 \times 10^{21} \mathrm{~kg}$, is required to be reconciled to $\sim 10^{20} \mathrm{~kg}$ by $\mathrm{Xu}, \mathrm{X} . \&$ Siegel, E. R[19]within the total solar system.

\section{Conclusions}

Sections $1-5$ show how a gravitationally bound vast rarified system of fixed ordinary matter like galaxy with low gravitational binding energy can store more matter energy over the relatively compact system. Since it has been presumed that both the of compact system and rarified system have same ordinary matter then from results in section-6, it is apparent for galaxy to be $90 \%$ more massive over the ordinary matter following equation(25) and describe the reason for flat rotation curve that fulfills the origin of hypothetical dark matter. But still the true nature and prevalence of the dark matter requires further adequate research and observations.

\section{References}

[1] Bardeen, M.J et al., Black Holes, Gordon and Breach Science Publisher, 2nd Printing ISBN0677156103 (1982) 222.

[2] Einstein, A. The Principle of Relativity, Dover Publication (1952), 148-150.

[3] Ferreira P. G., Starkmann, G. http://arxiv.org/abs/0911.1212 (2009).

[4] Flynn C. et al, MNRAS, 372, (2006)1158. http://dx.doi.org/10.1111/j.1365-2966.2006.10911.x.

[5] Garrett.K. and Duda, G. arXiv: 1006.2483v2 (2011).

[6] Gengel, R., Eisenhauer,F. \&Gillessen, S., Rev. Mod. Phys. 82, (2010) 3121. http://dx.doi.org/10.1103/RevModPhys.82.3121.

[7] Goldstein, H., Classical Mechanics, 2nd edition, Narosa Publishing House (1997) 82-85,308.

[8] Kormendy, J. \&Richstone, D. ARA\&A., 33, (1995) 605. http://dx.doi.org/10.1146/annurev.aa.33.090195.003053.

[9] Lo, K. Y. et al, Nature, 315, (1995)124L. http://dx.doi.org/10.1038/315124a0.

[10] Lyden, B. D. and Katz, J., MNRAS, 213, (1985) 22L.

[11] McMillan P. J. MNRAS, 414, (2011) 2454-2455. http://dx.doi.org/10.1111/j.1365-2966.2011.18564.x.

[12] Milgrom, M., ApJ, 270, (1983)365-370. http://dx.doi.org/10.1086/161130.

[13] Ohanian,H.C. http://arxiv.org/abs/1010.5557(2013)

[14] Rubin, V. et al,ApJ., 289(1985) 81-104. http://dx.doi.org/10.1086/162866.

[15] Rubin, V. et al, PNAS. 90, (1993)4814-4821. http://dx.doi.org/10.1073/pnas.90.11.4814

[16] Schödel, R. et al, Nature, 419(2002)494-496. http://dx.doi.org/10.1038/nature01121.

[17] Scott, D. et al http://arxiv.org/abs/astro-ph/0104435 (2001).

[18] Weinberg, S., Gravitation and cosmology. John Wiley and sons (1971), 152-155,635-636.

[19] Xu,X. Siegel, E. R. http://arxiv.org/abs/0806.3767 (2008).

[20] Zwicky, F., Ap.J, 86, 217-246 (1937). http://dx.doi.org/10.1086/143864. 